\title{
Physiologically Based Pharmacokinetic Modelling of Cytochrome P450 2C9-Related Tolbutamide Drug Interactions with Sulfaphenazole and Tasisulam
}

\author{
Everett J. Perkins $^{1}\left(\right.$ D $\cdot$ Maria Posada $^{1} \cdot$ P. Kellie Turner ${ }^{1} \cdot$ \\ Jill Chappell ${ }^{1} \cdot$ Wee Teck $\mathrm{Ng}^{2} \cdot$ Chris $^{\text {Twelves }}{ }^{3}$
}

Published online: 8 November 2017

(c) The Author(s) 2017. This article is an open access publication

\begin{abstract}
Background and Objectives Cytochrome P450 2C9 (CYP2C9) is involved in the biotransformation of many commonly used drugs, and significant drug interactions have been reported for CYP2C9 substrates. Previously published physiologically based pharmacokinetic (PBPK) models of tolbutamide are based on an assumption that its metabolic clearance is exclusively through CYP2C9; however, many studies indicate that CYP2C9 metabolism is only responsible for $80-90 \%$ of the total clearance. Therefore, these models are not useful for predicting the magnitude of CYP2C9 drug-drug interactions (DDIs). This paper describes the development and verification of SimCYP ${ }^{\circledR}$-based PBPK models that accurately describe the human pharmacokinetics of tolbutamide when dosed alone or in combination with the CYP2C9 inhibitors sulfaphenazole and tasisulam.

Methods A PBPK model was optimized in SimCYP ${ }^{\circledR}$ for tolbutamide as a CYP2C9 substrate, based on published in vitro and clinical data. This model was verified to replicate the magnitude of DDI reported with sulfaphenazole and was further applied to simulate the DDI with tasisulam, a small molecule investigated for the treatment of cancer. A clinical study (CT registration \# NCT01185548) was conducted in patients with cancer to assess the pharmacokinetic interaction of tasisulum with tolbutamide. A PBPK model was built for tasisulam, and
\end{abstract}

Everett J. Perkins

perkins_everett_j@lilly.com

1 Eli Lilly and Co, Indianapolis, IN, USA

2 Eli Lilly and Company, Singapore, Singapore

3 St James's Institute of Oncology, Leeds, UK the clinical study design was replicated using the optimized tolbutamide model.

Results The optimized tolbutamide model accurately predicted the magnitude of tolbutamide AUC increase (5.3-6.2-fold) reported for sulfaphenazole. Furthermore, the PBPK simulations in a healthy volunteer population adequately predicted the increase in plasma exposure of tolbutamide in patients with cancer (predicted AUC ratio $=4.7-5.4$; measured mean AUC ratio $=5.7$ ).

Conclusions This optimized tolbutamide PBPK model was verified with two strong CYP2C9 inhibitors and can be applied to the prediction of CYP2C9 interactions for novel inhibitors. Furthermore, this work highlights the utility of mechanistic models in navigating the challenges in conducting clinical pharmacology studies in cancer patients.

\section{Key Points}

A mechanism-based PBPK model is described for the prediction of CYP2C9-related drug interactions with tolbutamide. The model was verified with clinical data from the literature (sulfaphenazole) and from a drug interaction study with tasisulam in patients with cancer. This optimized tolbutamide PBPK model can be applied to the prediction of CYP2C9 interactions for novel inhibitors.

\section{Introduction}

Cytochrome P450 2C9 (CYP2C9) is a polymorphic enzyme responsible for the metabolism of many frequently used drugs, including warfarin, phenytoin, celecoxib, and sulfonylureas 
$[1,2]$. CYP2C9 expression is third highest among hepatic CYPs, accounting for an average of approximately $13 \%$ of the total CYP protein measured in human liver microsomes [3]. Individuals exhibiting the CYP2C9 poor metabolizer phenotype can be at increased risk of adverse events with drugs such as warfarin, and they often require lower doses of CYP2C9 substrates to be effective yet safe. Clinically significant drugdrug interactions (DDIs) have also been observed with CYP2C9 substrates, warfarin and phenytoin both being listed as narrow therapeutic index substrates [4].

Because of the potential effect of DDIs on the safety and efficacy of drugs, accurate prediction of potential drug interactions and pharmacogenetic effects for CYP substrates and inhibitors is important in drug development. Physiologically based pharmacokinetic (PBPK) modelling is increasingly applied for quantitative prediction of the pharmacokinetic effects of drug interactions and inter-individual variation. To this end, the commonly used software SimCYP ${ }^{\circledR}$ Simulator provides many predefined substrate and inhibitor models, with varying degrees of external verification. It is, however, important to continually reassess and modify PBPK models as additional physiological, in vitro, and clinical data become available. It is likewise important to verify such models for their intended purpose [5]. For example, it has been shown that PBPK models for patients with hepatic impairment have not been sufficiently developed, and physiologically based absorption models for neonates and infants need extensive verification [6, 7]. PBPK modelling has proven to be especially useful in oncology applications, where standard clinical pharmacology trials are not always feasible. In this manuscript, the development of a mechanistically based PBPK model for tolbutamide is described and verified for use in DDI prediction with two strong CYP2C9 inhibitors.

Tolbutamide is a first-generation oral sulfonylurea used for the treatment of people with diabetes since the $1950 \mathrm{~s}$ $[8,9]$. It is metabolized by CYP2C9 and is subject to drug interactions with CYP2C9 inhibitors [10]. Tolbutamide PBPK models have been used previously to verify intersystem extrapolation factors (ISEFs) for recombinant enzymes and to accurately model the effect of polymorphic enzyme expression in Chinese and Caucasian populations $[11,12]$. However, these models assumed that tolbutamide clearance was completely dependent on hepatic CYP2C9, and did not evaluate the effect of enzyme inhibition. The current SimCYP ${ }^{\circledR}$-provided sim-tolbutamide substrate model utilizes unique enzyme kinetic parameters for tolbutamide clearance. This default tolbutamide model has been partially characterized, with modifications described to better predict pharmacokinetics observed in Chinese individuals [11]. However, there are no published examples of accurate modelling of significant CYP2C9-related tolbutamide drug interactions using SimCYP ${ }^{\circledR}$.
Tasisulam is a novel anti-cancer agent that showed potential anti-angiogenic activity across a range of tumor types in animal and in vitro models. It was previously investigated in Phase II clinical studies in patients with melanoma, soft tissue sarcoma, and non-small cell lung cancer, but further development of this molecule has been terminated because of insufficient clinical benefit [13-17]. Tasisulam is highly bound to albumin $(>99.7 \%)$ and has a long terminal half-life (11 days) that approximates the half-life of circulating albumin [18]. In vitro studies showed tasisulam competitively inhibits CYP2C9, and could therefore affect the clearance and metabolism of sensitive CYP2C9 substrates, such as warfarin and tolbutamide [19].

The effect of tasisulam as an inhibitor of CYP2C9 on the clearance of tolbutamide was evaluated in a small Phase I study in patients with cancer, which is described in this report. However, the conduct of clinical pharmacology studies in patients with cancer patients is challenging, due to their health concerns, frequent concomitant medications, and issues around enrolment. As PBPK models are increasingly accepted by regulatory agencies as alternatives to clinical studies, further verification of the use of these models in specific disease states is needed [4, 5, 20]. This paper describes the optimization and verification of a mechanistically based middle-out PBPK model for tolbutamide based on published in vitro and in vivo studies, and then demonstrates the ability of the refined SimCYP ${ }^{\circledR}$ model to accurately predict tolbutamide interactions with both oral sulfaphenazole and intravenous tasisulam. These data support that this verified PBPK model can be successfully applied to predict interactions for other CYP2C9 inhibitors.

\section{Methods}

\subsection{PBPK Simulations}

All PBPK simulations were performed using PKPD profiles mode in $\operatorname{SimCYP}^{\circledR}$ Simulator $^{\mathrm{TM}}$ (v.15). Trial simulations were conducted using a 10 trial $\times 10$ subject design, with a healthy volunteer population (age 20-50; proportion of females $=0.5$ ) in the fasted state. All individuals in the population were set to be CYP2C9 extensive metabolizers to match the clinical studies. Default SimCYP ${ }^{\circledR}$ parameters were employed for models except where specifically noted.

\subsubsection{Tolbutamide}

A tolbutamide PBPK model was built using SimCYP ${ }^{\circledR}$ based on the Sim-tolbutamide model file with 
Table 1 Tolbutamide and sulfaphenazole PBPK input parameters

\begin{tabular}{|c|c|c|c|}
\hline \multicolumn{3}{|l|}{ Parameter } & \multirow[t]{2}{*}{ Source } \\
\hline Model & Tolbutamide & Sim-sulfaphenazole & \\
\hline Molecular weight (g/mol) & 270.3 & 314.36 & SimCYP $^{\circledR}$ Default \\
\hline $\log \mathrm{P}$ & 2.34 & 1.52 & SimCYP $^{\circledR}$ Default \\
\hline $\mathrm{p} K \mathrm{a}$ & 5.27 & 5.91 & SimCYP $^{\circledR}$ Default \\
\hline Blood to plasma ratio & 0.6 & 0.62 & SimCYP ${ }^{\circledR}$ Default \\
\hline$f_{\mathrm{u}}$ & 0.044 & 0.028 & $\operatorname{SimCYP}^{\circledR}$ Default \\
\hline $\mathrm{Fa}$ & 1 & 1 & SimCYP $^{\circledR}$ Default \\
\hline$k_{\mathrm{a}}\left(\mathrm{h}^{-1}\right)$ & 0.52 & 1.86 & SimCYP $^{\circledR}$ Default \\
\hline hPeff $\left(\times 10^{-4} \mathrm{~cm} / \mathrm{s}\right)$ & 0.95 & NA & SimCYP $^{\circledR}$ Default \\
\hline Hydrogen bond donors & 2 & 2 & SimCYP $^{\circledR}$ Default \\
\hline PSA $\left(A^{2}\right)$ & 83.65 & 98.39 & SimCYP $^{\circledR}$ Default \\
\hline $\mathrm{fu}_{\mathrm{Gut}}$ & 1 & 0.0275 & $\operatorname{SimCYP}^{\circledR}$ Default \\
\hline $\mathrm{Vd}_{\mathrm{ss}}(\mathrm{L} / \mathrm{kg})$ & 0.105 & 0.162 & SimCYP $^{\circledR}$ Default \\
\hline $\mathrm{CL}_{\mathrm{po}}(\mathrm{L} / \mathrm{h})$ & NA & 0.382 & SimCYP $^{\circledR}$ Default \\
\hline $\mathrm{CL}_{\text {renal }}(\mathrm{L} / \mathrm{h})$ & $0^{\mathrm{a}}$ & $0.084^{\mathrm{b}}$ & \\
\hline Distribution model & Minimal PBPK & Minimal PBPK & SimCYP $^{\circledR}$ Default \\
\hline CYP2C9 $\mathrm{CL}_{\text {int }}(\mu \mathrm{L} / \mathrm{min} / \mathrm{pmol}$ isoform $)$ & 0.065 & NA & $\begin{array}{l}\text { Retrograde calculation based on } \\
\text { an assumed CYP2C9 } \mathrm{fm} \text { of } 0.85\end{array}$ \\
\hline Additional HLM CL ( $\mu \mathrm{L} / \mathrm{min} / \mathrm{mg}$ protein) & 0.8 & NA & $\begin{array}{l}\text { Retrograde calculation based } \\
\text { on an assumed CYP2C } 9 \mathrm{fm} \text { of } 0.85\end{array}$ \\
\hline Ki CYP2C9 $(\mu \mathrm{M})$ & NA & 0.16 & SimCYP ${ }^{\circledR}$ Default \\
\hline $\mathrm{fu}_{\mathrm{mic}}$ & $1.0^{\mathrm{c}}$ & $0.972^{\mathrm{b}}$ & \\
\hline
\end{tabular}

$\log P$ partition coefficient, $p K a$ acid dissociation constant, $f_{u}$ fraction unbound in plasma, $F a$ fraction absorbed, $k_{a}$ absorption rate constant, $h P e f f$ effective permeability in humans, $N A$ not applicable, $P S A$ polar surface area, $f u_{G u t}$ fraction unbound in gut, $V d_{s s}$ volume of distribution at steady state, $C L_{p o}$ oral clearance, $C L_{i n t}$ intrinsic clearance, $H L M C L$ human liver microsomal clearance, $K i$ inhibition constant, $f u_{m i c}$ fraction unbound in microsomes, $P B P K$ physiologically based pharmacokinetic

${ }^{\text {a }}$ Set to zero

${ }^{\mathrm{b}} \mathrm{SimCYP}{ }^{\circledR}$ Default

${ }^{\mathrm{c}}$ Carlile et al. [51]

modifications. Model input parameters are listed in Table 1. The fraction metabolized (fm) was estimated to be approximately 0.85 , based on published clinical and in vitro data $[10,21-24]$. The CYP2C9 unbound intrinsic clearance was back-calculated from the IV clearance using the retrograde calculator in SimCYP, resulting in a value of $0.065 \mu \mathrm{L} / \mathrm{min} / \mathrm{pmol}$ of CYP isoform. A non-CYP2C9 hepatic metabolism clearance pathway was included in the elimination parameters to estimate approximately $15 \%$ of the total clearance.

\subsubsection{Sulfaphenazole-Tolbutamide Interaction}

To assess the predictive ability of the optimized tolbutamide model as a "victim" compound for DDIs, interaction studies were simulated utilizing the tolbutamide PBPK model described above and the default sim-sulfaphenazole model (Table 1). Performance verification has been conducted for the sim-sulfaphenazole model by Certara using published clinical data $[25,26]$. In the interaction, sulfaphenazole was defined as the CYP2C9 perpetrator and tolbutamide as the victim. Interaction simulations were conducted with the dosing paradigm previously described by Veronese et al., and the data from that study were used to verify the PBPK model [27]. Briefly, the trial design included a single tolbutamide dose administered $24 \mathrm{~h}$ after initiating sulfaphenazole dosing at $2000 \mathrm{mg}$ every $12 \mathrm{~h}$. Sulfaphenazole dosing continued for 4 days following tolbutamide administration. The trial duration was set to 20 days to capture the complete plasma concentration-time profile and 10,000 samples per individual were simulated.

\subsubsection{Tasisulam}

A minimal PBPK model was created for tasisulam using a combination of in vitro and clinically derived and predicted input values (Table 2). The dose of infused tasisulam was set at $40 \mathrm{mg} / \mathrm{kg}$ and the infusion time to $2 \mathrm{~h}$, approximating the dosing schedule of the JZAR clinical study described below. The plasma clearance of tasisulam was based on 
Table 2 Tasisulam PBPK input parameters

\begin{tabular}{lll}
\hline Parameter & Value & Source \\
\hline Molecular weight $(\mathrm{g} / \mathrm{mol})$ & 437.09 & \\
$\mathrm{LogP}$ & 3.8 & Measured in vitro \\
$\mathrm{p} K \mathrm{a}$ & 2.2 (acidic) & Measured in vitro \\
$\mathrm{Blood}$ to plasma ratio & 0.55 & Estimated value for an acid \\
$f_{\mathrm{u}}$ & 0.003 & Measured in vitro \\
$\mathrm{Vd}_{\mathrm{ss}}(\mathrm{L} / \mathrm{kg})$ & 0.165 & Gordon et al. [18] \\
$\mathrm{V}_{\mathrm{SAC}}(\mathrm{L} / \mathrm{kg})$ & 0.075 & Fitted from JZAR \\
$\mathrm{Q}_{\mathrm{SAC}}(\mathrm{L} / \mathrm{h})$ & 2.45 & Fitted from JZAR \\
$\mathrm{CL}$ systemic $(\mathrm{L} / \mathrm{h})$ & 0.025 & From clinical study results \\
$\mathrm{Ki} \mathrm{CYP} 2 \mathrm{C} 9(\mu \mathrm{M})$ & 0.1 & Measured in vitro \\
fu & 0.31 & Predicted with in-house QSAR model \\
Dosing route & $\mathrm{I} . \mathrm{V}$. infusion & Clinical data \\
Dose $(\mathrm{mg} / \mathrm{kg})$ & 40 & Clinical data \\
Dosing time $(\mathrm{h})$ & 2 & Clinical data
\end{tabular}

$\log P$ partition coefficient, $p K a$ acid dissociation constant, $f_{u}$ fraction unbound in plasma, $V d_{s s}$ volume of distribution at steady state, $V_{S A C}$ volume of single adjusting compartment, $Q_{S A C}$ blood flow of single adjusting compartment, $C L$ clearance, $K i$ inhibition constant, $f u_{m i c}$ fraction unbound in microsomes, $P B P K$ physiologically based pharmacokinetic observed clearance in patients. A single adjusting compartment (SAC) was included in the model to better capture the $C_{\max }$ and initial rapid distribution phase of tasisulam. The volume of distribution at steady state $\left(\mathrm{Vd}_{\mathrm{ss}}\right)$ was derived from clinical plasma concentration time profiles, using the Nelder-Mead minimization method and weighted-least squares as the objective function [28]. The values for the volume of distribution in the SAC and the intracompartmental clearance $(Q)$ were derived from the population pharmacokinetic analysis of tasisulam (unpublished data).

\subsubsection{Tasisulam-Tolbutamide Interaction}

Drug interaction trials were simulated utilizing the optimized tolbutamide and tasisulam PBPK models. The interaction was set at CYP2C9 with tasisulam as the perpetrator and tolbutamide as the victim, using the unadjusted measured inhibition constant (Ki) and predicted fraction unbound in the microsomes $\left(\mathrm{fu}_{\mathrm{mic}}\right)$ shown in Table 2. The interaction simulations were conducted with the dosing paradigm used in periods 2 and 3 of the JZAR study, described below. A fixed dose of $40 \mathrm{mg} / \mathrm{kg}$ and 2-h intravenous infusion of tasisulam was used to approximate the individualized dosing from this study. To simulate the acute interaction (Period 2), a $500 \mathrm{mg}$ oral dose of tolbutamide was introduced $2 \mathrm{~h}$ prior to the start of the tasisulam infusion. Simulation of the potential sustained effect of inhibition by tasisulam (Period 3) was conducted by introducing the $500 \mathrm{mg}$ tolbutamide dose $72 \mathrm{~h}$ after the start of the tasisulam infusion. The total duration of the trial simulations was 15 days, to adequately capture the AUC in the inhibited state.

\subsection{In vitro Inhibition CYP2C9 by Tasisulam}

The effect of tasisulam on CYP2C9 activity was assessed in vitro by measuring the inhibition of diclofenac $4^{\prime}$-hydroxylation in human liver microsomes. Incubation mixtures of approximately $500 \mu \mathrm{L}$ contained human hepatic microsomes $(0.05 \mathrm{mg} / \mathrm{mL}$ protein) in $100 \mathrm{mM}$ potassium phosphate buffer (pH 7.4), $1 \mathrm{mM} \mathrm{NADPH}$, and diclofenac $(0.5,5,10,15$, or $25 \mu \mathrm{M})$, in the absence or presence of $0.1,0.5,1$, or $2.5 \mu \mathrm{M}$ tasisulam as inhibitor. Following 4-min incubations at $37^{\circ} \mathrm{C}$, quenched incubations were analyzed for the formation of $4^{\prime}$-hydroxydiclofenac by LCMS/MS. 4'-Hydroxydiclofenac was obtained from Gentest Corporation (Woburn, MA, USA), diclofenac from Sigma Chemical Company (St. Louis, MO, USA) and human hepatic microsomes from CEDRA Corporation (Austin, TX, USA); all other reagents were purchased from commercial vendors.

Microsomal protein binding $\left(\mathrm{fu}_{\mathrm{mic}}\right)$ of tasisulam was estimated to be 0.31 using a proprietary quantitative structure-property relationship (QSPR) in silico model. This model was built based on the in vitro $\mathrm{Fu}_{\text {mic }}$ values for 7448 Eli Lilly library compounds, which were measured using equilibrium dialysis at $1 \mu \mathrm{M}$. The $\mathrm{Fu}_{\text {mic }}$ values in this training set ranged from 0.0001 to 1 (1st quartile- 0.28 ; median-0.54; 3rd quartile-0.72). The model was built using the support vector machine algorithm incorporating structural fingerprints [29], and the optimum fingerprint 
was identified as described previously by Desai et al. [30]. The model was evaluated using prospective validation wherein the predicted vs. measured $\mathrm{Fu}_{\text {mic }}$ values were compared for an additional 544 internal compound test set measured after the model was constructed. In this assessment, the predicted $\mathrm{Fu}_{\text {mic }}$ values were within twofold of the measured values for $78 \%$ compounds and within threefold for $95 \%$.

\subsection{Study JZAR: Tasisulam-Tolbutamide Interaction in Patients with Cancer}

\subsubsection{Eligibility Criteria}

Study H8K-MC-JZAR (JZAR, ClincialTrials.gov Registry \# NCT01185548) was conducted in patients to evaluate the effect of tasisulam on the pharmacokinetics of the CYP2C9 probe substrate tolbutamide. Eligible patients must have had histologically or cytologically confirmed solid malignancy or lymphoma with advanced and/or metastatic disease that was unresponsive to standard therapies and an ECOG score $\leq 1$. All patients had discontinued previous cancer therapies at least 30 days prior to enrolment and had recovered from any toxicities. Patients' serum albumin levels were required to be $\geq 30 \mathrm{~g} / \mathrm{L}$ (actual range $=39-48 \mathrm{~g} / \mathrm{L}$ ). Patients with documented brain metastases, leukemia, diabetes mellitus, and those receiving warfarin were excluded as were patients being treated with sulfonylureas. The severity of adverse events was assessed using the National Cancer Institute CTCAE version 4.02 [31].

\subsubsection{Study Design: Tasisulam Dosing and Pharmacokinetics}

Tasisulam was supplied as a sterile, lyophilized powder for IV infusion after reconstitution to a concentration of $50 \mathrm{mg} / \mathrm{mL}$ in sterile water and administered as an IV infusion over $2 \mathrm{~h}$ on Day 1 of a 28 day cycle. To provide an intravenous tasisulam dose within the exposure range that likely offered the best balance of efficacy and toxicity, a dosing-calculator that incorporated lean-body weight and pre-dose plasma albumin concentration was used in clinical trials [17, 18]. In Cycle 1, a loading dose of tasisulam was administered followed by continued dosing in subsequent cycles that was either 65 or $75 \%$ of the loading dose, depending on pre-cycle albumin levels.

Study JZAR was an open-label, fixed sequence, 3-period study. The CYP2C9 metabolizer status was determined for each patient. In each period, patients fasted for at least $8 \mathrm{~h}$ prior to dosing (except for water) and until at least $2 \mathrm{~h}$ after administration. To avoid cases of hypoglycaemia, all patients received $100 \mathrm{~g}$ of oral glucose $1 \mathrm{~h}$ after each dose of tolbutamide. In Period 1, patients received $500 \mathrm{mg}$ tolbutamide orally on Day 1, with an 8 day washout prior to starting Period 2. Periods 2 and 3 were 28 day cycles, with a single intravenous dose of tasisulam administered on Day 1. In Period 2, patients received a $500 \mathrm{mg}$ dose of tolbutamide orally, and then $2 \mathrm{~h}$ after tolbutamide administration they received a 2-h infusion of an individualized loading dose of tasisulam (as described above). The timing of administration of tolbutamide was selected so that the tolbutamide $T_{\max }$ (approximately $4 \mathrm{~h}$ ) coincided with that of tasisulam (i.e. end of infusion). In Period 3, the persistence of any potentially clinically relevant inhibitory effects of tasisulam on CYP2C 9 after $72 \mathrm{~h}$ was assessed by evaluating the pharmacokinetics of tolbutamide following a single 500-mg dose that was given $72 \mathrm{~h}$ after the tasisulam dose. Blood samples of approximately $2 \mathrm{~mL}$ were collected in Periods 1, 2, and 3 to determine the plasma concentrations of tolbutamide and tasisulam at the following times relative to the dosing (oral or start of infusion) of the respective drug:

\subsection{Tolbutamide}

Period 1: Pre-dose; and 0.5, 1, 1.5, 2, 2.5, 3, 4, 6, 8, 24, 48, and $72 \mathrm{~h}$.

Period 2: Pre-dose; and 0.5, 1, 1.5, 2, 2.5, 3, 4, 4.5, 5, 6, 8, 24, 48, 72, 120, 168, $336 \mathrm{~h}$.

Period 3: Pre-dose; and 0.5, 1, 1.5, 2, 2.5, 3, 4, 6, 8, 24, 48, 96 , and $264 \mathrm{~h}$.

\subsection{Tasisulam}

Period 2: Pre-infusion start; and 0.5, 1, 2, 2.5, 3, 4, 6, 22, 46, 70, 118, 166, and $334 \mathrm{~h}$.

Period 3: Pre-infusion start; and 1, 2, 2.5, 3, 4, 6, 48, 72, $72.5,73,73.5,74,74.5,75,76,78,80,96,120,168$, and $336 \mathrm{~h}$.

Following the drug interaction evaluation (Periods 1-3), patients could continue to receive tasisulam, at an individualized continuing dose, in 28 day cycles until evidence of disease progression, unacceptable toxicity or failure to meet minimum albumin levels required for dosing at which point the patients discontinued the study.

\subsubsection{Bioanalytical Methods}

All bioanalytical determinations of tasisulam and tolbutamide concentrations were conducted at Advion Bioservices, Inc., Ithaca, NY, USA. Plasma samples were analyzed for tasisulam concentrations using validated LC/ MS/MS methods for both high and low concentration ranges [32]. For the low range, the upper (ULQ) and lower limits of quantitation (LLQ) were 5 and $0.025 \mu \mathrm{g} / \mathrm{mL}$, 
respectively; for the high range, the ULQ and LLQ were 500 and $2.5 \mu \mathrm{g} / \mathrm{mL}$, respectively. Tolbutamide plasma concentration was quantified using a validated LC/MS/MS method. The LLQ was $1 \mathrm{ng} / \mathrm{mL}$ and the ULQ was $500 \mathrm{ng} /$ $\mathrm{mL}$.

\subsubsection{Pharmacokinetic Analysis Methods}

Pharmacokinetic parameters were determined from plasma concentrations using standard non-compartmental analysis techniques in WinNonlin (version 5.2; Certara, Princeton, NJ, USA). A mixed-effects model was used to analyze the $\log$-transformed tolbutamide $\mathrm{AUC}_{(0-\text { inf })}$ and $C_{\max }$. The model contained treatment (tolbutamide [reference], tolbutamide + tasisulam [test]); as the fixed effect and patient as a random effect. From the model, least squares mean (LSMean) and the 90\% CI for the difference of means were estimated, then transformed back to the original scale to estimate the ratio of geometric means and $90 \%$ CI for the comparison (tolbutamide + tasisulam versus tolbutamide alone). A nonparametric analysis of $T_{\max }$ was performed using the Wilcoxon signed rank test. Median differences between the treatments were calculated using the Hodges-Lehmann method. All calculations were performed using SAS $^{\circledR}$ software 9.1.

\section{Results}

\subsection{Tolbutamide and Sulfaphenazole PBPK}

PBPK simulations were conducted to evaluate the accuracy of predicting CYP2C9 DDI using tolbutamide as a substrate. Initial simulations using the default sim-tolbutamide and sim-sulfaphenazole input files dramatically over-predicted the reported $\mathrm{AUC}_{0-\text { inf }}$ ratio of approximately 5, with a mean predicted AUC ratio of $>27$ (data not shown). Therefore, a revised model was built with elimination parameters to better represent the current mechanistic understanding of tolbutamide clearance, as described in the Methods. The retrograde calculation function in SimCYP ${ }^{\circledR}$ was used to estimate the CYP2C9 Clint, with an additional nonspecific clearance pathway added to approximate the reported in vitro and in vivo observations. The resulting average fraction of tolbutamide metabolized (fm) by CYP2C9 was 0.81 in the overall simulated population. The sulfaphenazole interaction was then simulated to verify the optimized model, resulting in good reproduction of the reported tolbutamide pharmacokinetic parameters (Table 3; Fig. 1) in both inhibited and uninhibited states. The predicted AUC ratio of 5.7 was in line with that of 5.3 previously reported by Veronese [27]. A mean $C_{\max }$ value for tolbutamide was not reported from that clinical study; however, digitization of the representative plasma concentration curve included in the manuscript suggests values of approximately 39 and $52 \mathrm{mg} / \mathrm{L}$ in the uninhibited and inhibited states, respectively. Again, this is consistent with the simulated geometric mean $C_{\max }$ values of 39 and $53 \mathrm{mg} / \mathrm{L}$ in the uninhibited and inhibited states, respectively (Table 3 ).

\subsection{Pharmacokinetics}

\subsubsection{Study JZAR Patient Characteristics}

Four patients, one each with primary hepatic, renal, duodenal and esophageal cancer, received at least one dose of tolbutamide. The patients, three men and one woman, had ages ranging between 44 and 71 years. The mean weight was $73.2 \mathrm{~kg}$ (range 63.6-81.4). Only two patients completed the third period of the study. No CYP2C9 poor metabolizers enrolled in the study; all patients had $* 1 / * 1$ alleles.

\subsubsection{Tasisulam}

Figure 2 shows the mean plasma concentration versus time profiles in Period 2 and Table 4 summarizes the pharmacokinetic parameters of tasisulam from study JZAR, when intravenously infused over $2 \mathrm{~h}$. Following the end of infusion, plasma concentrations of tasisulam appeared to decline in a bi-phasic manner, with a long mean terminal half-life $\left(t_{1 / 2}\right)$ values of $248-274 \mathrm{~h}$. Likewise, the average clearance of tasisulam was slow and volume of distribution $\left(V_{\mathrm{z}}\right)$ low, at approximately $0.02 \mathrm{~L} / \mathrm{h}$ and $6.6-8.3 \mathrm{~L}$, respectively. These values are in line with those previously reported for tasisulam in patients with cancer [17, 18]. Although tasissulam concentrations were quantifiable prior to dosing in Period 3, the tasisulam dosing algorithm yielded similar plasma concentration-time curves and pharmacokinetic parameters in Periods 2 and 3 (Table 4).

\subsubsection{Tolbutamide}

When tolbutamide was administered without tasisulam, tolbutamide plasma concentrations appeared to decline in a monophasic manner (Fig. 3, Table 5). Following co-administration of tolbutamide with tasisulam in Periods 2 and 3 , the $T_{1 / 2}$ of tolbutamide was considerably longer, with mean values of 43 and $35 \mathrm{~h}$, respectively, compared to a mean $T_{1 / 2}$ of $7 \mathrm{~h}$ when administered alone (Period 1). Individual tolbutamide $t_{1 / 2}$ values ranged from 5 to 9,34 to 55 and 29 to $42 \mathrm{~h}$, respectively, for Periods 1, 2, and 3. Apparent total plasma clearance of tolbutamide decreased following tasisulam administration, with mean values decreasing from $1.1 \mathrm{~L} / \mathrm{h}$ in Period 1 to $0.19 \mathrm{~L} / \mathrm{h}$ in Periods 2 
Table 3 Simulated and reported tolbutamide pharmacokinetics following an oral dose of $500 \mathrm{mg}$, alone or following multiple doses of $2000 \mathrm{mg}$ sulfaphenazole every $12 \mathrm{~h}$

\begin{tabular}{|c|c|c|c|c|}
\hline & \multicolumn{2}{|l|}{ Tolbutamide } & \multicolumn{2}{|l|}{ Tolbutamide (+ sulfaphenazole) } \\
\hline & Simulated GM (\%CV) & Reported mean (SD) & Simulated GM (\%CV or CI) & Reported mean (SD) \\
\hline $\mathrm{CL}_{\mathrm{po}}(\mathrm{L} / \mathrm{h})$ & $0.84(62 \%)$ & $0.86(0.3)^{\mathrm{a}} ; 0.82(0.11)^{\mathrm{b}}$ & $0.15(48 \%)$ & $0.16(0.04)^{\mathrm{b}}$ \\
\hline$C_{\max }(\mathrm{mg} / \mathrm{L})$ & $38.8(35 \%)$ & $39^{\#, \mathrm{~b}}$ & $53.0(35 \%)$ & $52^{\#, \mathrm{~b}}$ \\
\hline $\mathrm{AUC}(\mathrm{mg} \cdot \mathrm{h} / \mathrm{L})$ & $585(53 \%)$ & $586.8(52.2)^{\mathrm{b}}$ & $3359(40 \%)$ & $3100(1044)^{\mathrm{b}}$ \\
\hline AUC ratio & NA & NA & $5.74(5.3,6.22)$ & $5.3^{\mathrm{b}}$ \\
\hline$C_{\max }$ ratio & NA & AN & $1.37(1.33,1.4)$ & $1.33^{\#, \mathrm{~b}}$ \\
\hline
\end{tabular}

$S D$ standard deviation, $C L_{p o}$ oral clearance, $C_{\max }$ maximal plasma concentration, $A U C$ area under the curve, $N A$ not applicable, $G M$ geometric mean, $C V$ coefficient of variation

References: ${ }^{\mathrm{a}}[52] ;{ }^{\mathrm{b}}[27]$

${ }^{\#}$ Estimated from digitized graph of representative subject
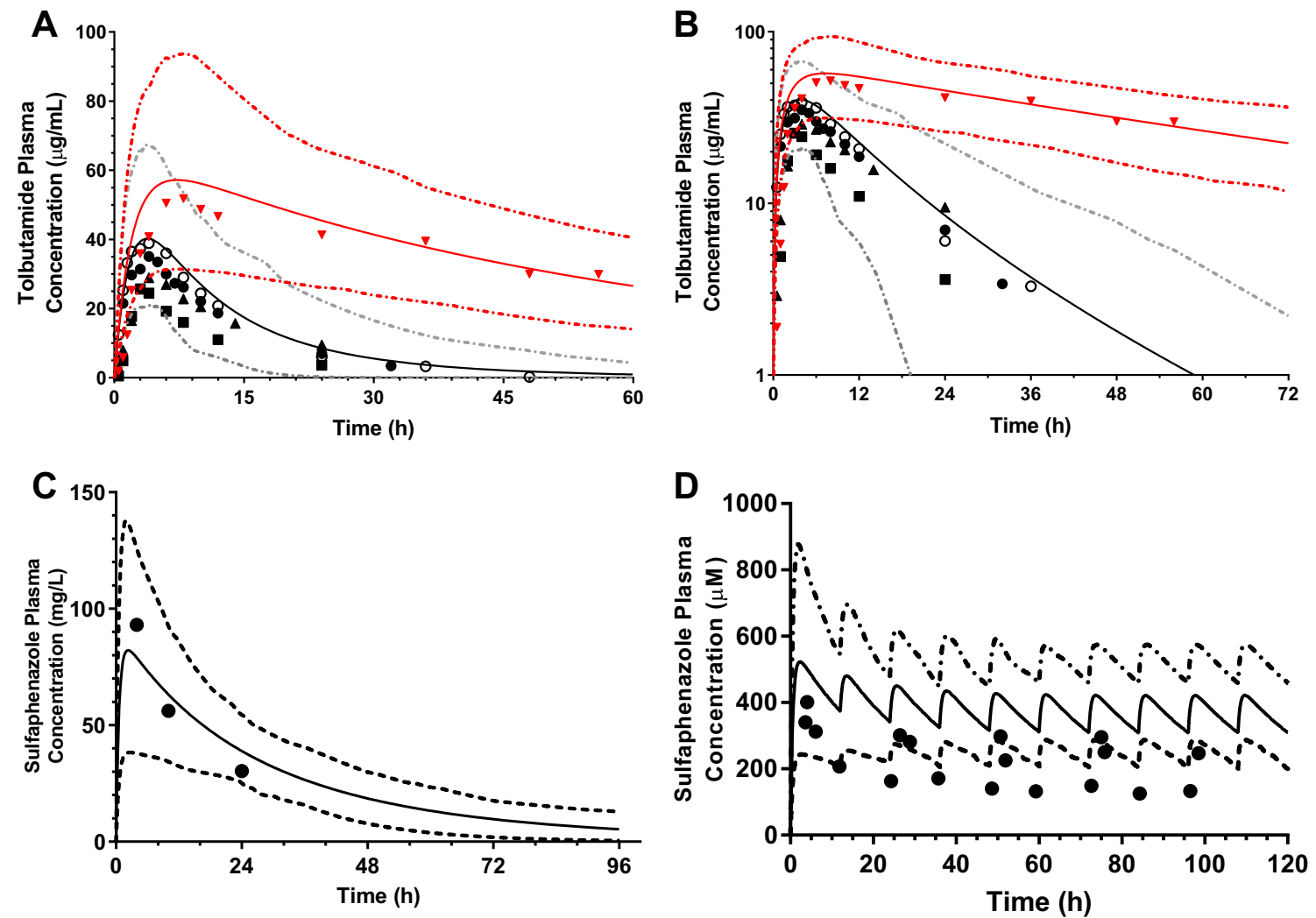

Fig. 1 Simulated and reported plasma concentration-time profiles of tolbutamide alone (black) and in the presence of sulfaphenazole (red), after an oral $500 \mathrm{mg}$ dose in healthy subjects a linear and $\mathbf{b}$ log-linear scale. Solid lines represents the predicted mean concentrations of tolbutamide. Dotted lines represent the predicted 5th and 95th percentiles. Solid squares represent the mean reported data from Nordmark et al. [39]. The solid circles represent the mean plasma concentrations from Madsen et al. [38]. The open circles and red triangles represent the concentrations from Veronese et al. [27]. The solid black triangles represent the mean concentrations from Dixit and Rao [40]. c Simulated and reported plasma concentration-time

profiles of sulfaphenazole after a single oral $1000 \mathrm{mg}$ dose. Solid black line represents the predicted mean concentration of sulfaphenazole. Dotted black lines represent the predicted 5th and 95th percentiles. Solid circles represent reported data by Ries et al. [26]. d Simulated and reported plasma concentration-time profiles of sulfaphenazole after an oral dose of $2000 \mathrm{mg}$ followed by $500 \mathrm{mg}$ twice daily for 4 days. Solid black line represents the predicted mean concentration of sulfaphenazole. Dotted black lines represent the predicted 5th and 95th percentiles. Solid circles represent reported data by Bunger et al. [25] 


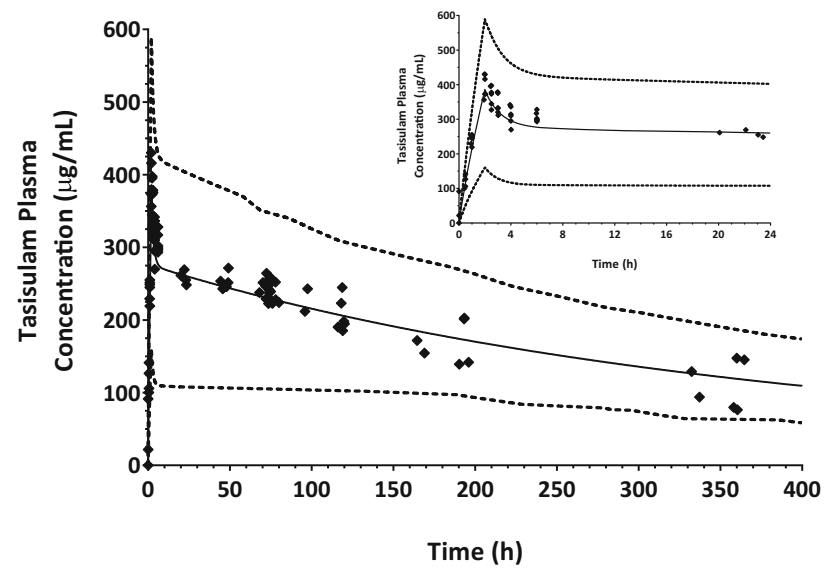

Fig. 2 Observed and predicted plasma concentration time profiles for tasisulam (Study JZAR Period 2). Points represent the observed data. Solid lines represent the predicted mean tasisulam concentration. Dotted lines represent the predicted 5th and 95th percentiles of simulation. Inset shows detail of early time points

and 3. In contrast, there appeared to be no change in the apparent volume of distribution between periods. Therefore, co-administration of tasisulam and tolbutamide resulted in an increase in the systemic exposure $\left(\mathrm{AUC}_{0-\text { tlast }}\right)$ of tolbutamide $(2650 \mathrm{mg} \cdot \mathrm{h} / \mathrm{L})$ in Period 2 compared to administration alone $(467 \mathrm{mg} \cdot \mathrm{h} / \mathrm{L})$ in Period 1, with a ratio of Least Squared Means of 5.70 (90\% CI $4.74,6.86)$. The PK parameters for tolbutamide were very similar in Period 2 and Period 3, confirming that the long half-life of tasisulam resulted in prolonged inhibition (at least $72 \mathrm{~h}$ ) of CYP2C9. The $C_{\max }$ for tolbutamide was similar between periods (Table 5 ).

\subsubsection{Clinical Safety}

The most frequent treatment emergent adverse event was dizziness, which occurred in two patients. One patient who experienced dizziness $1.5 \mathrm{~h}$ into the tasisulam infusion in Period 2 had a blood glucose at baseline of $83 \mathrm{mg} / \mathrm{dL}$
( $4.6 \mathrm{mmol} / \mathrm{L}$ ) and by $1.5 \mathrm{~h}$ into the infusion this had fallen to $59 \mathrm{mg} / \mathrm{dL}$ ( $3.3 \mathrm{mmol} / \mathrm{L})$, but rose again within $5 \mathrm{~min}$ of consuming food; this patient again experienced dizziness on Day 9 of Period 2. The second patient experienced dizziness on Day 13 of Period 2.

One patient had an $\mathrm{AE}$ of thrombocytopenia considered related to study treatment that started in Period 2 as Grade 2 (Day 8), increased to Grade 3 (Day 9) and then Grade 4 (Day 10) before returning to Grade 2 (Day 12) and Grade 1 at follow-up (Day 32). All other AEs were Grade 1 or Grade 2 and there were no deaths during the study. One serious adverse event (CTCAE Grade 1 musculoskeletal chest pain resulting in hospitalization) was reported that the investigator considered unrelated to study treatment.

\subsection{Tasisulam DDI Simulation}

The PBPK model for tasisulam adequately reproduced in simulated healthy subjects the plasma concentration-time profiles of tasisulam observed in cancer patients (described above) after an infusion of $40 \mathrm{mg} / \mathrm{kg}$ of tasisulam as seen in Fig. 2. The model also reproduced $C_{\max }$ and AUC values in plasma, with predicted:observed ratios of 1.09 and 1.03, respectively (Table 4). The observed plasma concentration-versus-time profile for tolbutamide in the presence and absence of tasisulam are well described by the PBPK models and the in vitro inhibition parameters. The corresponding observed and predicted AUC and $C_{\max }$ ratios of tolbutamide in the presence of tasisulam are shown in Table 5. All predicted values are within $20-25 \%$ from the observed values.

\section{Discussion}

This paper describes the development and verification of a SimCYP ${ }^{\mathrm{TM}}$-based PBPK model that accurately describes human pharmacokinetic drug interactions of tolbutamide,
Table 4 Simulated and observed (Study JZAR) tasisulam pharmacokinetics following a 2-h intravenous infusion of tasisulam

\begin{tabular}{|c|c|c|c|}
\hline & \multicolumn{3}{|c|}{$\begin{array}{l}\text { Tasisulam } \\
\text { Geometric mean (\%CV or range) }\end{array}$} \\
\hline & Simulated & Observed-Period $2(N=4)$ & Observed-Period $3(N=2)$ \\
\hline $\mathrm{CL}(\mathrm{L} / \mathrm{h})$ & $0.02(32 \%)$ & $0.02(31 \%)$ & 0.02 \\
\hline$C_{\max }(\mathrm{mg} / \mathrm{L})$ & $409(37 \%)$ & $393(9 \%)$ & 403 \\
\hline $\mathrm{AUC}_{0-t}(\mathrm{mg} \cdot \mathrm{h} / \mathrm{L})$ & $70,417(28 \%)$ & $62,600(11 \%)$ & 68,200 \\
\hline$V_{\mathrm{z}}(\mathrm{L})$ & $\mathrm{NC}$ & $8.3(16)$ & 6.6 \\
\hline$T_{\max }(\mathrm{h})$ & $2(2-2)$ & $1.99(1.93-2.00)$ & $2.25(2.00-2.50)$ \\
\hline$T_{1 / 2}(\mathrm{~h})$ & $287(88-679)$ & $274(177-398)$ & $248(178-345)$ \\
\hline
\end{tabular}

$\% C V$ coefficient of variation, $C L$ clearance, $C_{\max }$ maximal plasma concentration, $A U C_{0-t}$ area under the curve from 0 to last time point, $N C$ not calculated, $V_{z}$ volume of distribution, $T_{\max }$ time of maximal concentration, $T_{1 / 2}$ half-life 

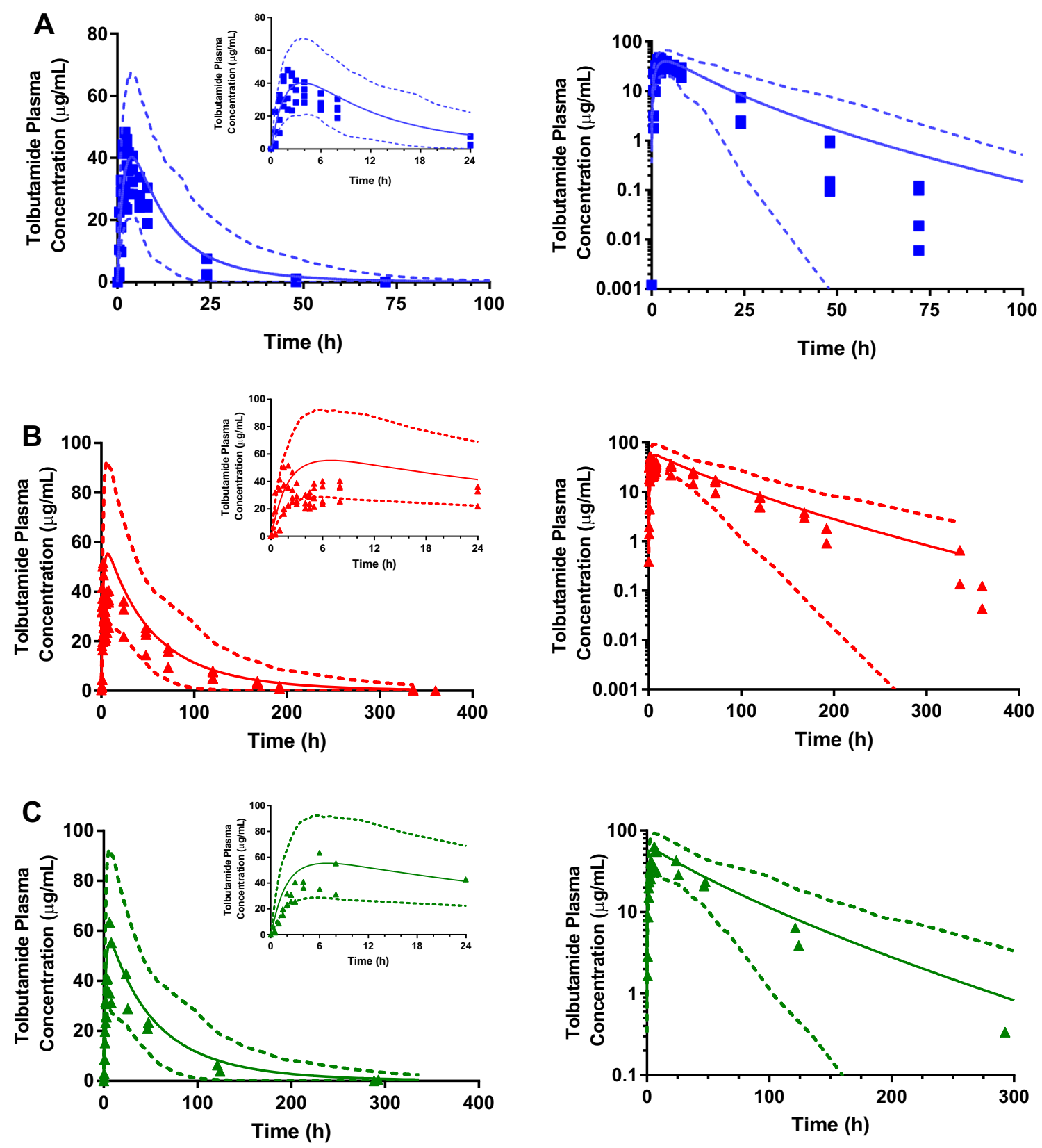

Fig. 3 a Observed and predicted plasma concentration-time profiles of tolbutamide in the absence of tasisulam (Period 1, blue). Solid points represent the observed tolbutamide data from study JZAR. Solid lines represent the predicted mean concentration of tolbutamide. Dotted lines represent the predicted 5th and 95th percentiles. Inset shows detail of early time points. b Observed and predicted plasma concentration-time profiles of tolbutamide in the presence of tasisulam (Period 2, red). Solid points represent the observed tolbutamide data from study JZAR. Solid lines represent the predicted

in this case when dosed alone or in combination with the CYP2C9 inhibitors sulfaphenazole and tasisulam.

Tolbutamide is extensively metabolized in humans, with $85 \%$ of an oral dose excreted as metabolites in the urine over $48 \mathrm{~h}$ [33]. Tolbutamide metabolism occurs predominantly through oxidation to 4-hydroxytolbutamide (4-HT),

mean concentration of tolbutamide in the presence of tasisulam (Period 2). Dotted lines represent the predicted 5th and 95th percentiles. Inset shows detail of early time points. c Observed and predicted plasma concentration-time profiles of Tolbutamide in the presence of tasisulam (Period 3, green). Solid points represent the observed tolbutamide data from study JZAR. Solid lines represent the predicted mean concentration of tolbutamide in the presence of tasisulam (Period 3). Dotted lines represent the predicted 5th and 95th percentiles. Inset shows detail of early time points

which is further oxidized to carboxytolbutamide [34-36]. The initial rate-limiting hydroxylation step is catalyzed by CYP2C9. As a result, tolbutamide exhibits polymorphic pharmacokinetics and is affected by concomitant CYP2C9 inhibitors, such as sulfaphenazole [2, 22, 37]. Although tolbutamide has been extensively studied, and is an FDA 
Table 5 Simulated and observed tolbutamide pharmacokinetics following a 2-hour intravenous infusion of tasisulam and $500 \mathrm{mg}$ oral dose of tolbutamide administered $2 \mathrm{~h}$ prior (Period 2) or $72 \mathrm{~h}$ following (Period 3) the start of infusion

\begin{tabular}{|c|c|c|c|c|c|c|}
\hline & \multicolumn{2}{|c|}{$\begin{array}{l}\text { Tolbutamide } \\
\text { GM (\% CV or range) }\end{array}$} & \multicolumn{2}{|c|}{$\begin{array}{l}\text { Tolbutamide } \\
(+ \text { Tasisulam-Period } 2) \\
\text { GM }(\% \mathrm{CV} \text { or range })\end{array}$} & \multicolumn{2}{|c|}{$\begin{array}{l}\text { Tolbutamide } \\
\text { (+ Tasisulam-Period 3) } \\
\text { GM (range) }\end{array}$} \\
\hline & Simulated & Observed & Simulated & Observed $(N=4)$ & Simulated & Observed $(N=2)$ \\
\hline $\mathrm{CL}_{\mathrm{po}}(\mathrm{L} / \mathrm{h})$ & $0.84(62 \%)$ & $1.07(29 \%)$ & $0.14(49 \%)$ & $0.19(20 \%)$ & $0.15(49 \%)$ & 0.19 \\
\hline$C_{\max }(\mathrm{mg} / \mathrm{L})$ & $38.9(35 \%)$ & $39.6(26 \%)$ & $52.7(35 \%)$ & $39.8(18 \%)$ & $52.6(35 \%)$ & 48.0 \\
\hline $\mathrm{AUC}_{0-t}(\mathrm{mg} \cdot \mathrm{h} / \mathrm{L})$ & $594(54 \%)$ & $467(29 \%)$ & $3433(42 \%)$ & $2650(19 \%)$ & $3375(41 \%)$ & 2690 \\
\hline $\mathrm{Vd}_{\mathrm{ss}} / F(\mathrm{~L})$ & $8.1(27 \%)$ & $10.7(21 \%)$ & $8.1(27 \%)$ & $11.5(30 \%)$ & $8.1(27 \%)$ & 9.28 \\
\hline$T_{\max }(\mathrm{h})$ & $4.0(3.8-4.2)$ & $2.25(2.0-3.0)$ & $6.9(3.5-11.0)$ & $5.0(1.5-8.0)$ & $6.9(3.5-11.0)$ & 5.0 \\
\hline$T_{1 / 2}(\mathrm{~h})$ & $7.0(1.6-22)$ & $6.9(5.4-8.5)$ & $31.6(6.2-113)$ & $42.7(33.9-54.5)$ & $31.6(6.2-113)$ & $34.7(29.1-41.5)$ \\
\hline AUC ratio & NA & NA & $5.8(5.4-6.2)^{\mathrm{a}}$ & $5.7(4.74-6.86)$ & $5.7(5.3-6.1)$ & 5.8 \\
\hline$C_{\max }$ ratio & NA & NA & $1.35(1.32-1.38)^{\mathrm{a}}$ & $1.0(0.77-1.32)$ & $1.35(1.32-1.39)$ & 1.2 \\
\hline
\end{tabular}

$\% C V$ coefficient of variation, $C L_{p o}$ oral clearance, $C_{\max }$ maximal plasma concentration, $A U C_{0-t}$ area under the curve from 0 to last time point, $V d_{s s} / F$ apparent oral volume of distribution, $T_{\max }$ time of maximal concentration, $T_{1 / 2}$ half-life, $N A$ not applicable, $G M$ geometric mean

${ }^{\mathrm{a}}$ Value in parentheses for AUC and $C_{\max }$ ratios is $90 \%$ confidence interval

recommended in vivo substrate for investigating CYP2C9 DDI, relatively few clinical interaction studies have been published, all of which were in healthy subjects [38-41].

One of the most significant tolbutamide interactions has been observed with concomitant oral administration of sulfaphenazole [27, 37, 42]. To assess the utility of PBPK modelling to predict CYP2C9 DDI, an initial investigation of the default SimCYP ${ }^{\circledR}$ tolbutamide (sim-tolbutamide) model was conducted using the software-provided parameters for tolbutamide and sulfaphenazole. The clinical trial design described by Veronese et al. [27] was replicated in SimCYP, but with a larger population of virtual subjects. In this study, a single oral dose of tolbutamide $(500 \mathrm{mg})$ was administered to healthy volunteers following multiple doses of sulfaphenazole. These simulations appeared to adequately reproduce the individual plasma concentration profiles for both drugs, but using these compound files in a simulated interaction trial resulted in a more than fivefold difference between predicted and reported clinical values. This suggests that while the default sim-tolbutamide model can reproduce the pharmacokinetics of tolbutamide alone, the model's high dependence on CYP2C9 for tolbutamide clearance might be inaccurate.

The sim-tolbutamide model assumes CYP2C9 $\mathrm{fm}=1$, but there are several lines of evidence suggesting that a more accurate value is between 0.8 and 0.9 . An early paper reported that approximately $85 \%$ of an oral tolbutamide dose was excreted as hydroxy- and carboxy-metabolites in $48 \mathrm{~h}$ by healthy human volunteers [33]. Similar results were reported in 1990, with $51-96 \%$ of the dose excreted as these two CYP2C9-dependent metabolites in $24 \mathrm{~h}$ [34]. A more recent ${ }^{14} \mathrm{C}$ microdose study in healthy subjects also produced similar findings of $77 \%$ of the radioactive dose recovered in the urine of $\mathrm{CYP} 2 \mathrm{C} 9 * 1 / * 1$ individuals as these metabolites over $72 \mathrm{~h} \mathrm{[43].} \mathrm{The} \mathrm{total} \mathrm{recovery} \mathrm{of} \mathrm{the}$ ${ }^{14} \mathrm{C}$ microdose in this group was $<85 \%$ and only about $1 \%$ was recovered as parent tolbutamide in the urine, leaving open the possibility that other minor metabolic pathways are involved in its systemic clearance.

Multiple in vitro and computational approaches to quantitatively assess the role of CYP2C9 in tolbutamide clearance have also been described, which together indicate an $\mathrm{fm}$ in the range of 0.7-0.9, with possible additional contribution by CYP2C19 [1, 21-24, 35, 44]. However, pharmacogenetic studies in humans have not revealed a significant influence of CYP2C19 polymorphisms on the clearance of tolbutamide in vivo [45-47]. Although these studies could not rule out a minor role of CYP2C19, the focus of the current studies is on tolbutamide as a CYP2C9 probe substrate. Therefore, to account for the non-CYP2C9 component of clearance, an additional hepatic clearance component was included in the model to approximate $15 \%$ of the total average clearance, but it was not assigned specifically to CYP2C19. The retrograde calculator was used to estimate the remaining CYP2C9-mediated clearance, with a resulting population mean $\mathrm{fm}$ of 0.82 , which is consistent with the range of reported estimates described above. The revised model parameters reproduced the plasma pharmacokinetic profile adequately (Fig. 1). Using this revised set of input parameters, the sulfaphenazole interaction simulation was re-run, resulting in a predicted AUC ratio (Table 3) consistent with the value reported by Veronese et al. [27].

Evaluating drug interactions in patients with cancer presents several challenges in addition to specific requirements that may arise due to a drug's unique posology or 
pharmacokinetics. Due to the safety profile of tasisulam and its stage of clinical development, DDI studies were restricted to patients with advanced cancer for whom life expectancy may be short. To be eligible for the study, patients were required to have a life expectancy of at least 1-2 months to complete the minimum required sampling in two study periods. The advanced cancer patient population also presented a design challenge with respect to the need to administer a probe drug in the absence of potentially active treatment. As the delay between enrolment and starting tasisulam was a maximum of 8 days, this proved acceptable to investigators, patients, and ethics committees. In addition, patients with advanced cancer often take many concomitant medications. However, to be able to interpret the drug interaction data, concomitant medications involved in CYP2C9 pathways were excluded, presenting another challenge to study design and enrolment.

Despite the hurdles to study design and conduct, the tasisulam-tolbutamide interaction study clearly demonstrated that tasisulam is a strong CYP2C9 inhibitor. A challenge in designing these studies was the lack of data on variability of substrates in patients with cancer. However, the large effect size observed provided a conclusive answer despite the limited sample size for the CYP2C9 study with tolbutamide. Therefore, information from a small number of patients proved extremely valuable in informing the clinical development program. This might not be the case for drugs with smaller predicted effect sizes, such as weak or moderate CYP inhibitors.

The limitations on conducting extensive DDI studies in patients with cancer highlight the value of PBPK models to simulate clinical studies and refine clinical trial designs. In fact, a majority of PBPK references in recent drug approvals are related to anti-cancer drugs [5]. For a drug, such as tasisulam, an accurate prediction of the magnitude of DDI and resulting pharmacokinetic profile can both ensure an adequate study design and help extrapolate clinical data to a broader population or alternate dose regimens. To illustrate this point, a top-down minimal PBPK model was constructed for tasisulam and subsequently combined with the optimized tolbutamide model in DDI simulations. The tasisulam PBPK model in a simulated healthy population adequately reproduced the PK observed after intravenous dosing in cancer patients, which is characterized by a rapid initial distribution phase followed by a long terminal half-life related to its low intrinsic clearance and tight binding to plasma proteins. Furthermore, replicating the JZAR clinical design in SimCYP ${ }^{\circledR}$ resulted in accurate prediction of the observed fivefold increase in tolbutamide AUC. It is notable that the clearance of tolbutamide in the uninhibited state was slightly higher in the JZAR patients than reported values in healthy volunteers, but the significance of this observation is unknown. While the simulated trials predicted a $30 \%$ increase in $C_{\max }$, no change in tolbutamide $C_{\max }$ was observed in this study; however, the measured concentrations were within the lower 95th percentile of the predicted values. It should also be noted that the patients included in study JZAR were selected to have serum albumin levels $>30 \mathrm{~g} / \mathrm{L}$, resulting in plasma protein concentrations within the range of normal values included in the simulated healthy population. Since albumin concentrations are often decreased in some patients with advanced cancer and both tolbutamide and tasisulam are highly bound drugs, this would need to be considered if extrapolating to a more heterogeneous patient population [48]. Oncology-specific SimCYP $^{\circledR}$ population models have been described that account for observed differences in plasma proteins, age, CYP expression, etc., $[48,49]$. Such models might serve as a useful framework for further predictions.

\section{Conclusion}

A middle-out PBPK model for tolbutamide was optimized and verified for the prediction of CYP2C9-mediated DDI in SimCYP. This model effectively reproduces the human plasma pharmacokinetic profile of tolbutamide and accurately predicts the magnitude of exposure changes when co-administered with both oral and intravenous CYP2C9 inhibitors. The prediction of the observed fivefold increase in tolbutamide AUC with co-administration of tasisulam in cancer patients highlights the utility of mechanistic models in navigating the challenges associated with conducting clinical pharmacology studies in cancer patients. In the current example, the simulated healthy volunteer population appeared to be applicable to the small cancer patient group studied. However, additional work is needed to better understand the effect of different cancer disease states on CYP-dependent drug clearance in larger populations [50].

Acknowledgements This research was supported by NIHR Leeds Clinical Research Facility. The authors thank Basira Abdul for review of the modelling data; Jennifer Gillespie for conduct of in vitro CYP inhibition studies; Prashant Desai for description of the $\mathrm{fu}_{\text {mic }}$ in silico model; and Duncan Wilkins, Rebecca Kristeleit, and Alan Anthoney for their roles in the conduct of clinical study JZAR.

\section{Compliance with Ethical Standards}

Ethical compliance Study JZAR was conducted according to applicable laws and regulations, good clinical practices and the Declaration of Helsinki. Informed consent was obtained from each patient before study participation, and the study protocol was approved by the appropriate ethical review board (Leeds [West] Research Ethics Committee, Leeds, UK).

Funding This work was sponsored by Eli Lilly and Company. 
Conflicts of interest Drs. Perkins, Posada, Chappell, Turner, and $\mathrm{Ng}$ were employees and stockholders of Eli Lilly and Company during the conduct of this work and/or preparation of this manuscript.

Open Access This article is distributed under the terms of the Creative Commons Attribution-NonCommercial 4.0 International License (http://creativecommons.org/licenses/by-nc/4.0/), which permits any noncommercial use, distribution, and reproduction in any medium, provided you give appropriate credit to the original author(s) and the source, provide a link to the Creative Commons license, and indicate if changes were made.

\section{References}

1. Miners JO, Birkett DJ. Cytochrome P4502C9: an enzyme of major importance in human drug metabolism. Br J Clin Pharmacol. 1998;45(6):525-38.

2. Zhou K, Donnelly L, Burch L, Tavendale R, Doney AS, Leese G, et al. Loss-of-function CYP2C9 variants improve therapeutic response to sulfonylureas in type 2 diabetes: a Go-DARTS study. Clin Pharmacol Ther. 2010;87(1):52-6. https://doi.org/10.1038/ clpt.2009.176.

3. Achour B, Russell MR, Barber J, Rostami-Hodjegan A. Simultaneous quantification of the abundance of several cytochrome P450 and uridine $5^{\prime}$-diphospho-glucuronosyltransferase enzymes in human liver microsomes using multiplexed targeted proteomics. Drug Metab Dispos. 2014;42(4):500-10. https://doi.org/ 10.1124/dmd.113.055632.

4. United States Food and Drug Administration. Guidance for industry, drug interaction studies - study design, data analysis, implications for dosing, and labeling recommendations. 2012.

5. Yoshida K, Budha N, Jin JY. Impact of physiologically based pharmacokinetic models on regulatory reviews and product labels: frequent utilization in the field of oncology. Clin Pharmacol Ther. 2017;101(5):597-602. https://doi.org/10.1002/cpt. 622.

6. Sinha V, Zhao P, Huang SM, Zineh I. Physiologically based pharmacokinetic modeling: from regulatory science to regulatory policy. Clin Pharmacol Ther. 2014;95(5):478-80. https://doi.org/ 10.1038/clpt.2014.46.

7. Yu G, Zheng Q-S, Li G-F. Similarities and differences in gastrointestinal physiology between neonates and adults: a physiologically based pharmacokinetic modeling perspective. AAPS J. 2014;16(6):1162-6. https://doi.org/10.1208/s12248-014-9652-1.

8. Craig JW, Miller M. The use of tolbutamide (orinase) in the management of various types of diabetes mellitus and studies of possible mechanisms of its action. Metabolism. 1956;5(6 Part 2):868-74.

9. Miller WL Jr, Dulin WE. Orinase, a new oral hypoglycemic compound. Science. 1956;123(3197):584-5.

10. Miners JO, Birkett DJ. Use of tolbutamide as a substrate probe for human hepatic cytochrome P450 2C9. Methods Enzymol. 1996;272:139-45.

11. Barter ZE, Tucker GT, Rowland-Yeo K. Differences in cytochrome P450-mediated pharmacokinetics between chinese and caucasian populations predicted by mechanistic physiologically based pharmacokinetic modelling. Clin Pharmacokinet. 2013;52(12):1085-100. https://doi.org/10.1007/s40262-0130089-y.

12. Crewe HK, Barter ZE, Yeo KR, Rostami-Hodjegan A. Are there differences in the catalytic activity per unit enzyme of recombinantly expressed and human liver microsomal cytochrome P450 2C9? A systematic investigation into inter-system extrapolation factors. Biopharm Drug Dispos. 2011;32(6):303-18. https://doi. org/10.1002/bdd.760.

13. Hamid O, Ilaria R Jr, Garbe C, Wolter P, Maio M, Hutson TE, et al. A randomized, open-label clinical trial of tasisulam sodium versus paclitaxel as second-line treatment in patients with metastatic melanoma. Cancer. 2014;120(13):2016-24. https://doi.org/ $10.1002 /$ cncr. 28635

14. Jotte RM, Von Hoff DD, Braiteh F, Becerra CR, Richards DA, Smith DA, et al. An innovative, multi-arm, complete phase $1 \mathrm{~b}$ study of the novel anti-cancer agent tasisulam in patients with advanced solid tumors. Invest New Drugs. 2015;33(1):148-58. https://doi.org/10.1007/s10637-014-0160-z.

15. Kirkwood JM, Gonzalez R, Reintgen D, Clingan PR, McWilliams RR, de Alwis DP, et al. A phase 2 study of tasisulam sodium (LY573636 sodium) as second-line treatment for patients with unresectable or metastatic melanoma. Cancer. 2011;117(20):4732-9. https://doi.org/10.1002/cncr.26068.

16. Scagliotti GV, Ilaria R Jr, Novello S, von Pawel J, Fischer JR, Ermisch S, et al. Tasisulam sodium (LY573636 sodium) as thirdline treatment in patients with unresectable, metastatic non-smallcell lung cancer: a phase-II study. J Thorac Oncol. 2012;7(6):1053-7. https://doi.org/10.1097/JTO. 0b013e3182519d79.

17. Ryan CW, Matias C, Agulnik M, Lopez-Pousa A, Williams C, de Alwis DP, et al. A phase II study of tasisulam sodium (LY573636 sodium) as second-line or third-line treatment for patients with unresectable or metastatic soft tissue sarcoma. Invest New Drugs. 2013;31(1):145-51. https://doi.org/10.1007/s10637-012-9819-5.

18. Gordon MS, Ilaria R, de Alwis DP, Mendelson DS, McKane S, Wagner MM, et al. A phase I study of tasisulam sodium (LY573636 sodium), a novel anticancer compound, administered as a 24-h continuous infusion in patients with advanced solid tumors. Cancer Chemother Pharmacol. 2013;71(1):21-7. https:// doi.org/10.1007/s00280-012-1917-8.

19. Twelves CJ, Wilkins DK, Anthoney A, Chappell J, Ng WT, Turner PT, et al. P06.06 Effects of tasisulam sodium on the pharmacokinetics of tolbutamide in patients with advanced solid tumours. Ann Oncol. 2013;24(1):i34-i. https://doi.org/10.1093/ annonc/mdt048.5.

20. European Medicines Agency, Committee for Medicinal Products for Human Use (CHMP). Guideline on the qualification and reporting of physiologically based pharmacokinetic (PBPK) modelling and simulation-draft. 2016.

21. Brown HS, Ito K, Galetin A, Houston JB. Prediction of in vivo drug-drug interactions from in vitro data: impact of incorporating parallel pathways of drug elimination and inhibitor absorption rate constant. Br J Clin Pharmacol. 2005;60(5):508-18. https:// doi.org/10.1111/j.1365-2125.2005.02483.x.

22. Castellan AC, Tod M, Gueyffier F, Audars M, Cambriels F, Kassai B, et al. Quantitative prediction of the impact of drug interactions and genetic polymorphisms on cytochrome P450 2C9 substrate exposure. Clin Pharmacokinet. 2013;52(3):199-209. https://doi.org/10.1007/s40262-013-0031-3.

23. McGinnity DF, Tucker J, Trigg S, Riley RJ. Prediction Of CYP2C9-mediated drug-drug interactions: a comparison using data from recombinant enzymes and human hepatocytes. Drug Metab Dispos. 2005;33(11):1700-7. https://doi.org/10.1124/dmd. 105.005884 .

24. Soars MG, Gelboin HV, Krausz KW, Riley RJ. A comparison of relative abundance, activity factor and inhibitory monoclonal antibody approaches in the characterization of human CYP enzymology. Br J Clin Pharmacol. 2003;55(2):175-81.

25. Bünger $P$, Diller W, Führ J, Krüger-Thiemer E. Vergleichende Untersuchungen an neueren Sulfanilamiden. Arzneimittelforschung. 1961;11(3):247-55. 
26. Riess W, Schmid K, Keberle H. Über den Stoffwechsel von Sulfaphenazol $\quad\left(\right.$ Orisul $\left.{ }^{\circledR}\right)$. Klinische Wochenschrift. 1965;43(13):740-4.

27. Veronese ME, Miners JO, Randles D, Gregov D, Birkett DJ. Validation of the tolbutamide metabolic ratio for population screening with use of sulfaphenazole to produce model phenotypic poor metabolizers. Clin Pharmacol Ther. 1990;47(3):403-11.

28. Nelder JA, Mead R. A simplex method for function minimization. Comp J. 1965;7(4):308-13. https://doi.org/10.1093/comjnl/ 7.4.308.

29. Erickson JA, Mader MM, Watson IA, Webster YW, Higgs RE, Bell MA, et al. Structure-guided expansion of kinase fragment libraries driven by support vector machine models. Biochim Biophys Acta. 2010;1804(3):642-52. https://doi.org/10.1016/j. bbapap.2009.12.002.

30. Desai PV, Raub TJ, Blanco MJ. How hydrogen bonds impact $P$ glycoprotein transport and permeability. Bioorg Med Chem Lett. 2012;22(21):6540-8. https://doi.org/10.1016/j.bmcl.2012.08.059.

31. Cancer Therapy Evaluation Program. Common terminology criteria for adverse events (Version 4.02).

32. Simon GR, Ilaria RL, Sovak MA, Williams CC, Haura EB, Cleverly AL, et al. A phase I study of tasisulam sodium (LY573636 sodium), a novel anticancer compound in patients with refractory solid tumors. Cancer Chemother Pharmacol. 2011;68(5):1233-41. https://doi.org/10.1007/s00280-011-1593-0.

33. Thomas RC, Ikeda GJ. The metabolic fate of tolbutamide in man and in the rat. J Med Chem. 1966;9(4):507-10.

34. Miller AK, Adir J, Vestal RE. Excretion of tolbutamide metabolites in young and old subjects. Eur J Clin Pharmacol. 1990;38(5):523-4.

35. Lee CR, Pieper JA, Frye RF, Hinderliter AL, Blaisdell JA, Goldstein JA. Tolbutamide, flurbiprofen, and losartan as probes of CYP2C9 activity in humans. J Clin Pharmacol. 2003;43(1):84-91.

36. Veronese ME, McManus ME, Laupattarakasem P, Miners JO, Birkett DJ. Tolbutamide hydroxylation by human, rabbit and rat liver microsomes and by purified forms of cytochrome P-450. Drug Metab Dispos. 1990;18(3):356-61.

37. Pond SM, Birkett DJ, Wade DN. Mechanisms of inhibition of tolbutamide metabolism: phenylbutazone, oxyphenbutazone, sulfaphenazole. Clin Pharmacol Ther. 1977;22(5 Pt 1):573-9.

38. Madsen H, Enggaard TP, Hansen LL, Klitgaard NA, Brøsen K. Fluvoxamine inhibits the CYP2C9 catalyzed biotransformation of tolbutamide. Clin Pharmacol Ther. 2001;69(1):41-7. https://doi. org/10.1067/mcp.2001.112689.

39. Nordmark A, Andersson A, Baranczewski P, Wanag E, Ståhle L. Assessment of interaction potential of AZD2066 using in vitro metabolism tools, physiologically based pharmacokinetic modelling and in vivo cocktail data. Eur J Clin Pharmacol. 2014;70(2):167-78. https://doi.org/10.1007/s00228-013-1603-8.
40. Dixit AA, Rao YM. Pharmacokinetic interaction between diltiazem and tolbutamide. Drug Metab Drug Interact. 1999;15(4):269-77.

41. Teng R, Mitchell P, Butler K. Evaluation of the pharmacokinetic interaction between ticagrelor and tolbutamide, a cytochrome P450 2C9 substrate, in healthy volunteers. Int J Clin Pharmacol Ther. 2013;51(4):305-12. https://doi.org/10.5414/CP201749.

42. Back DJ, Tjia J, Monig H, Ohnhaus EE, Park BK. Selective inhibition of drug oxidation after simultaneous administration of two probe drugs, antipyrine and tolbutamide. Eur J Clin Pharmacol. 1988;34(2):157-63.

43. Ikeda T, Aoyama S, Tozuka Z, Nozawa K, Hamabe Y, Matsui T, et al. Microdose pharmacogenetic study of (1)(4)C-tolbutamide in healthy subjects with accelerator mass spectrometry to examine the effects of CYP2C9 $* 3$ on its pharmacokinetics and metabolism. Eur J Pharm Sci. 2013;49(4):642-8. https://doi.org/ 10.1016/j.ejps.2013.05.016.

44. Komatsu K, Ito K, Nakajima Y, Kanamitsu S, Imaoka S, Funae $\mathrm{Y}$, et al. Prediction of in vivo drug-drug interactions between tolbutamide and various sulfonamides in humans based on in vitro experiments. Drug Metab Dispos. 2000;28(4):475-81.

45. Kirchheiner J, Bauer S, Meineke I, Rohde W, Prang V, Meisel C, et al. Impact of CYP2C9 and CYP2C19 polymorphisms on tolbutamide kinetics and the insulin and glucose response in healthy volunteers. Pharmacogenetics. 2002;12(2):101-9.

46. Miners J. CYP2C9 polymorphism: impact on tolbutamide pharmacokinetics and response. Pharmacogenetics. 2002;12(2):91-2.

47. Shon JH, Yoon YR, Kim KA, Lim YC, Lee KJ, Park JY, et al. Effects of CYP2C19 and CYP2C9 genetic polymorphisms on the disposition of and blood glucose lowering response to tolbutamide in humans. Pharmacogenetics. 2002;12(2):111-9.

48. Cheeti S, Budha NR, Rajan S, Dresser MJ, Jin JY. A physiologically based pharmacokinetic (PBPK) approach to evaluate pharmacokinetics in patients with cancer. Biopharm Drug Dispos. 2013;34(3):141-54. https://doi.org/10.1002/bdd.1830.

49. Thai H-T, Mazuir F, Cartot-Cotton S, Veyrat-Follet C. Optimizing pharmacokinetic bridging studies in paediatric oncology using physiologically-based pharmacokinetic modelling: application to docetaxel. Br J Clin Pharmacol. 2015;80(3):534-47. https://doi.org/10.1111/bcp.12702.

50. Coutant DE, Kulanthaivel P, Turner PK, Bell RL, Baldwin J, Wijayawardana SR, et al. Understanding disease-drug interactions in cancer patients: implications for dosing within the therapeutic window. Clin Pharmacol Ther. 2015;98(1):76-86. https:// doi.org/10.1002/cpt.128.

51. Carlile DJ, Hakooz N, Bayliss MK, Houston JB. Microsomal prediction of in vivo clearance of CYP2C9 substrates in humans. Br J Clin Pharmacol. 1999;47(6):625-35.

52. Scott J, Poffenbarger PL. Pharmacogenetics of tolbutamide metabolism in humans. Diabetes. 1979;28(1):41-51. 